\title{
Managing Conversational Streams By Explorative Mind-Maps
}

\author{
Jayanta Poray, Christoph Schommer \\ Department of Computer Science and Communication \\ University Luxembourg \\ 6, Rue Coudenhove-Kalergi, L-1359 Luxembourg \\ Email: \{jayanta.poray, christoph.schommer\}@uni.lu
}

\begin{abstract}
In this paper, we introduce an explorative but adaptive-associative information management system in the presence of a natural conversation. We take advantage of these explorative mind-maps, which have been demonstrated in [10] and which are altogether a management framework that emerges automatically from the data input stream it gets. An explorative mind-map is a non-verificative but dynamic system that basis on the natural paradigm: it changes its complexity continuously and fosters symbolic cells according to internal activation states. Generally, the structure mirrors a mental state where the oblivion of associated facts arrive once the stimulation decreases. As a special case, immortal mind-maps may be taken with respect to conversation streams, where the "die off" and the "forget" of a stimulated input may be disregarded but exploited. Considering two mind-maps $A_{1,2}$ and $B_{1,2}$ for two conversational partners $\boldsymbol{A}$ and $B$, then a mind-map $*_{1}$ represents the self-conversation and $*_{2}$ the conversational stream of the conversational partner. If we merge these mind-maps, we may apply the out-coming results for the computation of trust.

Index Terms-Cognitive Systems, Brain Informatics, Bioinspired Learning, Adaptive Information Management, Trustworthiness.
\end{abstract}

\section{Problem Statement}

Assume that Alice and $B o b$ talk to each other by a natural conversation. Then both Alice and Bob receive a conversational signals from the conversational partner. Both independently then decide about its relevance: textual patterns inside the conversational streams become extracted or even not, they are summarised and kept in mind - or even handled as noise. If both Alice and Bob store conversational signals, then both will know something more about the conversing partner. And, Alice surely estimates a certain belief about $B o b$ and her own believes with respect to the subject of the conversation (and vice versa).

With respect to the question on how Alice and Bob manage these signals, we understand the corresponding management system as an associative and flexible structure that emerges from the input it gets. We call this structure an explorative mind-map (Chapter II; Figures 1 and 3). Assume that Alice and $B o b$ own such individual mind-maps that foster on their mental status and on the other's conversed input (Figure 1). Then, both Alice and Bob store temporal patterns inside their own mental images about the conversational partner and are able to merge their own mind-maps (representing what they think and believe) with the own mind-map representing the conversing partner.

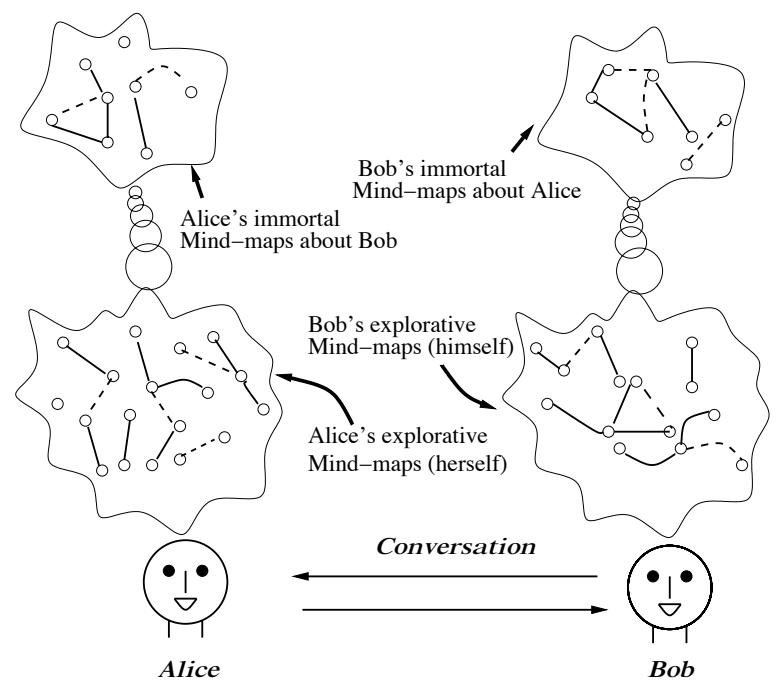

Fig. 1. A sample conversation between Alice and Bob : both share their own (immortal) mind-map as well as a mind-map about the conversing partner.

Our challenge is now to find a suitable way to merge the mind-maps and to come up with a consistent and reliable mind-map structure. Furthermore, explorative mind-maps include a forget factor and therefore risk that parts of the mind-map get lost if they are not activated regularly. At a first glance, this is a disadvantage, since a conversed content should be present all the time and may not forgotten. In case that an important information occurs at the beginning of a conversation but is never communicated again, then this would consequently lead to a decrease of connections and cells, and finally to a forget of this content. Redundant information is kept out and regarded as noise, but a strict consideration of each stream input is generally seen as infeasible. Moreover, there exists always a certain capacity and limitation regarding the harvesting of information.

We then come up with a model compromise and design each person's own mind-map including the continuously forgetful states. With respect to the information collection of the conversed person, however, and since conversations are generally not being that intensive, we favour immortal mindmaps, but additionally come up with the occasional application 
of cleaning, which occurs in case that the mind-map reaches its highest capacity. For example, cells and connections, which do not have a necessary strength, are automatically removed. The advantage of this approach is that both all information is recorded and and the natural example remains assured.

\section{Architecture of A Mind-MAP}

In this chapter, we describe the fundamental architecture of explorative mind-maps. We accent that explorative mindmaps rely on the natural principle on sensations and the corresponding propagation of stimuli to a final destination in order to process streams of symbolic data.

\section{A. Explorative mind-maps}

Explorative mind-maps share a principle through an associative architecture that incrementally processes accepted symbolic stimuli to a consistent informational structure. This is similar to the natural paradigm, especially connectionist approaches like [4], [5], [9], but on contrast to a verifying processing of a user's thoughts, the explorative mind-maps are built from the bottom up, meaning that a mind-map's existence exclusively depend on each other by incoming signals. Explorative mind-maps share a sub-symbolic architecture that is composed of interacting entity cells. As mentioned above for the natural principle, these cells foster on a processing of symbolic data streams and a stimulation/inhibition-principle of adjacent connections. The activation of such a connectionist architecture bases on a dynamic construction of cell structures during the processing of the input stream.
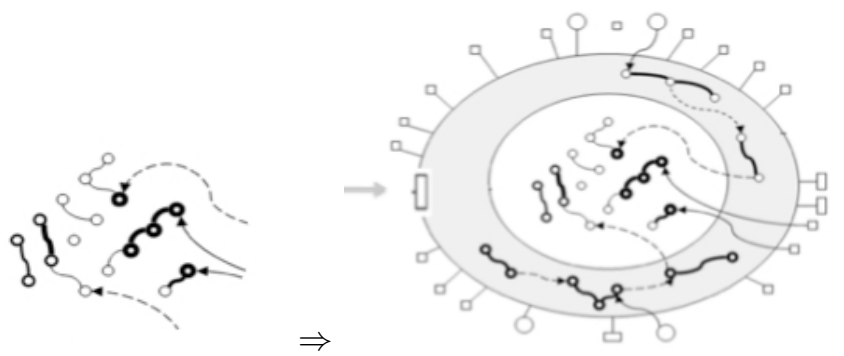

Fig. 2. A clump of conversational cells (left) that become established during the conversation and that are finally sent to explorative mind map (right). At first, it is placed to the short term memory (grey area) but possibly being sent to the long term (inner core) memory.

In Figure 3 we observe a mini-network (clump of cells) contains three patterns, namely $A, B$ and $C$. In a filtration phase the cells $\mathrm{A}$ and $\mathrm{C}$ enter inside the short-term memory and finally merge with the long-term memory, where the unused pattern $B$ kick-out from the mind-map. The bottom-up process of merging cells together (see section III) starts with a stimulation phase, where a stimulating stream data is absorbed by receptor (input) cells $i_{k}$, which then decompose the stream to its entities. For example, a text stream is decomposed to its word entities, transactional streams to item entities, and so on. Using filter cells $f_{k}$, exactly those receptor cells $i_{k}$ are inhibited if they do not address a semantic interest. In the mini-network phase, the collections of entities, which occur at such a specific time-point, form a mini-network with fully connected entity cells $e_{k}$. A mind-map merger starts once the mini-network is established: here, the mini-network is sent to the mind-map and is merged with the existing entity cell in the mind-map (initially, the mind-map is empty). In this regard, the specified merge references an action of mini-network and entity cells - that share a same representation - to a unique entity cell $e_{k}$. The activation status of an entity cell is then increased in case it has been merged. If two adjacent entity cells $e_{k}$ and $e_{l}$ of the mind-map are activated by the mininetwork at the same time, their connection weight $\omega\left(e_{k}, e_{l}\right)$ is increased by the Hebbian learn rate $\phi$. If two adjacent entity cells $e_{k}$ and $e_{l}$ remain inactivated, a fragment $\delta$ decreases their connection weight. The association becomes forgotten if the connection is below the activation threshold $\epsilon_{\min }$. The mind-map may degenerate if due to less intensive stimuli. In a final phase, the communication with the outside, especially the informing about the actual state with regard to contents, is realised. This is to be done either on demand - the user explicitly sends a request - or unsolicited.

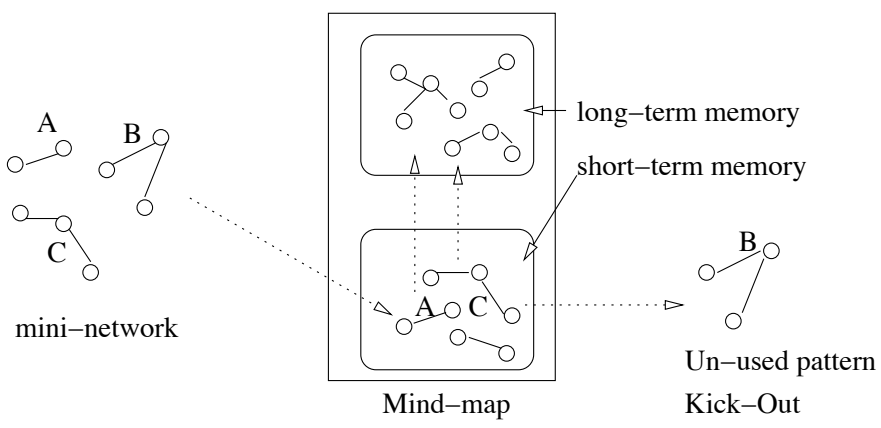

Fig. 3. The architectural design of an explorative mind-map with mininetwork, short-term and long-term memory.

Insofar, the consequence of the brief descriptions above is not only that explorative mind-maps are non-deterministic regarding its size, appearance, and communication but that explorative mind-maps stand for a life-cycle process that depends on the intensity of incoming stimulations and the activity inside the mind-map. Before the corresponding mini-networks arrive on the left side, they become firstly assigned to the receptor cells, become then filtered, and finally being put to the mini-network structure. This mini-network is sent to the inner core - which represents the mind-map management system and then merged with the mind-map itself (inner area). An extension to the explorative mind-maps are cognitive mindmaps, which we call as to be explorative like the mind-maps described above but that additionally use temporal mining components to detect temporal changes inside the skeletons. As a consequence, both the short term memory and the longterm memory may benefit from such an additional information. Also, the mind-map becomes more artificially human, meaning that a temporal analysis of data streams can be seen as an intellectual (artificial) intelligence. On the other side, an alternative to explorative mind-maps are immortal mind-maps. In contrast to the given mind-maps, they do never support 
a slightly decrease of activations or cells (in the sense of forgetting something) but simply remain present once they have occurred.

\section{B. Short-Term and Long-Term Memory}

The mind-map is managed by two types of memories. The short-term memory stands - in analogy to the human example - for a physical and mental place of storing/managing entities like impressions, word associations, etc. In the evolutionary beginning, it is empty but will grow up to a certain size the more and more it receives an input. And indeed, our explorative mind-map model fosters the short-term memory as the place where collections of entity cells as well as skeletons of cells (see section II-D) may be stored (in case that they are interesting and worth). The Short-Term Memory has a limited capacity $\chi$, a temperature $\tau$ within an interval $\left[\epsilon_{\min }, \ldots, \epsilon_{\max }\right]$, and the transfer parameter $\nu_{p}$ to send longer-established (or worth) cell-based patterns to the Long-Term Memory (longterm memory). The long-term memory is similar to the shortterm memory but is more a static place (once a skeleton is there, it is rather impossible to get rid of it), being a communication platform with the external environment (users, other systems).

\section{Mind-map Threshold Factors}

A unit is a pattern that is constructed with both the entity cells and the associated connections. Such units change from time to time with their inherent charge (activation value). The total amount of charge in some time slice is the measure of energy for a pattern. Moreover, we consider the capacity $\chi$ as a binary variable that directly depends on a set of binary boundary thresholds parameters $C_{1}, C_{2}$, and $C_{3}$ with

$$
\begin{aligned}
& C_{1}=\left\{\begin{array}{cc}
1 & \text { if } \\
0 & \text { else }
\end{array} \quad \theta_{\min } \leq n \leq \theta_{\max }\right. \\
& C_{2}=\left\{\begin{array}{cc}
1 & \text { if } \\
0 & \text { else }
\end{array} \zeta_{\min } \leq m \leq \zeta_{\max }\right. \\
& C_{3}=\left\{\begin{array}{cc}
1 & \text { if } \\
0 & \text { else }
\end{array} \quad \epsilon_{\min } \leq \tau \leq \epsilon_{\max }\right.
\end{aligned}
$$

where $n$ is the number of patterns inside a mind-map and $m$ the number of units inside a pattern. The complexity $\tau$ $\left(=\frac{\left|e_{i}\right|}{\left|c_{i}\right|}\right)$ is defined as the ratio between the number of entity cells $\left(e_{i}\right)$ and the number of their associated connections $\left(c_{i}\right)$. If the number of connections is higher than the number of entity cells, then the temperature $\tau$ of the mind-map will be significantly higher as well. This is a symptom of complex patterns. These types of patterns need to be simplified for maintain the healthy state of the mind-map. With this, we define the capacity as

$$
\chi=\prod_{i=1}^{3} C_{i}
$$

If $\chi=0$, then the capacity reaches its maximum limit. Operations are applicable only if $\chi$ switches to 1 .

\section{Skeletons}

In the context of pattern formation process the skeletons are the higher-activated clump of entity cells that occur temporally or frequently and that consist of entity cells sharing a strong activity; skeletons have been stable over a period of time or are recurrent in a sense that they occur again after being lost. As mentioned in section II-B, the skeletons are sent to the shortterm memory and/or long-term memory. As for the natural example, skeletons may be impressions or notices, which are to be discovered: depending on its state and temporal eligibility, existing skeletons may stay inside the short-term memory or be sent to the long-term memory. And, once being in the longterm memory, skeletons can be communicated to the external ad may become applied: for example, a knowledge center that contains content of a high relevance.

The process of identifying such skeletons is a comprehensive task that will be continuously done by e.g. sequential pattern algorithms, which accepts a sequence candidate in case it merges the frequency threshold. To send such a sequence to the short-term memory, some parameters must be taken into account:

- Density: how dense is the sequence in respect to the time? If it is stable or recurrent with at most a certain time gap $\gamma$, then it is accepted; otherwise, it is not.

- Size: how large is the sequence? Often, sequences of length $l_{s} \leq 3$ may occur, which are probably necessary but even not expressive enough.

- Balance: the activation balance $\pi$ of all participating cells may differ, probably, in a too divergent way. Therefore, homogeneous sequences could probably be accepted, whereas heterogeneous patterns stay outside.

\section{E. Membranes}

It may appear that entity cells are sometimes unlike to themselves although they contain the same subject. This may occur - for example - in the presence of speeches when words refer to different meanings (ambiguity). For example, although the content of an entity cell $e_{i}=\{$ ball $\}$ is - at first glance identical to another entity cell $e_{j}=\{$ ball $\}$, the meaning of it may be different since ball both stands for dance evening and a seating. Figure 4 represents the situation, where the entity cell $A$ tries to get merged with the already existing entity cell $A$. To enrich each entity cell and to avoid a wrongly merge in general, we protect each cell by a membrane. The membrane is a "coat of logic" that controls the correct similarity among the cells (see chapter III). The membrane of a cell is permeable in case that both associated entity cells refer to the same meaning (otherwise not). 


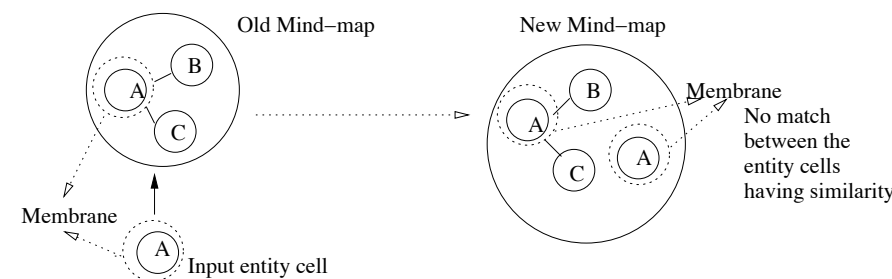

Fig. 4. Identical entity cells that are protected by membranes.

\section{F. Cold and Overheating}

A merge of cells, skeletons, and/or mind-maps leads us to one fundamental problem: an uncontrolled complexity of a mind-map $M$ in the presence of a given mind-map capacity $\chi$. With uncontrolled complexity we refer to two situations where in the first case a too low number of cells and/or skeleton exist and in the second the number of cells and skeletons exceeds the given capacity limit. We call these two situations a cold and overheating of the mind-map. Given a set $E$ of entity cells $e_{k}$, then a mind-map $M$ is healthy in case that the temperature $\tau$ inside the mind-map $M$ is within a normal range:

$$
\epsilon_{\min } \leq \tau \leq \epsilon_{\max }
$$

The mind-map $M$ is called to be diseased in case that the temperature $\tau$ is either too low (cold) or too high (overheated):

$$
\tau \leq \epsilon_{\min } \vee \tau \geq \epsilon_{\max }
$$

Keeping mind-maps into a diseased state yields to a mindmap of non-operating cells (this is both for entity cells $e_{k}$, filter cells $e_{k}$, and receptor cells $i_{k}$ ). Any merge can not take place because the adaptors remain frozen or fluid. As a matter of self-regulation, counteractions are available: fever in the sense of increasing the mind-map temperature and cooling in the sense of decreasing the mind-map temperature. With this, each mind-map ends in a consistent (healthy) state through this self-regulation process by fever and cooling.

\section{G. Pattern Transfer Rate}

The transfer of the temporal patterns to the short-term memory and from the short-term memory to the long-term memory depends on several conditions: temperature $\tau$, Energy $\eta$, a d Capacity $\chi$. If the mind-map is healthy $\left(\epsilon_{\min } \leq \tau \leq \epsilon_{\max }\right)$ then the memories keep in their active state of pattern transfer. But when the temperature exceeds $\epsilon_{\max }$ or falls below $\epsilon_{\min }$, then the transfer process stops until it refresh to the healthy state. During healthy state the best skeletons are sent to the short-term memory and/or long-term memory, respectively. The energy $\eta_{p}$ inside the pattern $p$ can be counted as the total amount of its inherent activation value, where $m$ is the number of entity cells and their associated connections and $a_{i}$ the corresponding activation value:

$$
\eta_{p}=\sum_{i=1}^{m} a_{i}
$$

This energy level can be considered as the most influencing factor to identify the healthy pattens, present inside the shortterm memory. The patterns, having minimum energy threshold $\left(\eta_{p}^{\text {min }}\right)$ are eligible to enter into the long-term memory. Also the best candidates among the healthy patterns normally contain high energy. These best patterns are separately distinguished as skeletons, where $\eta_{p}^{s}$ is the skeleton energy threshold:

$$
\eta_{p}^{s}>\eta_{p}^{\min }
$$

The volume of mind-map measures by the number of patterns and the corresponding entity cells with their connections. The goal is to consistent growth of the mind-map with the healthy patterns. In this regard, the capacity $(\chi)$ of the mind-map is taken into consideration, which is a combined factor of mind-map parameters, including the volume and the temperature. The transfer of the healthy patterns from short-term memory to long-term memory is influenced by the capacity with the observed threshold parameters.

We come with the transfer rate $\nu_{p}$ (from short-term memory to long-term memory) for the pattern $p$, which depends on its energy $\left(\eta_{p}\right)$ along with the current state of capacity $(\chi)$ of the mind-map:

$$
\nu_{p}=\chi \times \eta_{p}
$$

If $\chi=0$ then the transfer rate $\nu_{p}=0$ as well. However, if $\chi=1$, then a gradual value will become assigned to $\nu_{p}$. Furthermore, when the mind-map is in a healthy state (i.e., $\chi=1$ ) the overall Transfer Rate $\nu$ could be computed as the sum of all transfer rates for $n$ patterns.

$$
\nu=\sum_{p=1}^{n} \nu_{p}
$$

In case of a diseased mind-map state, the transfer stops.

\section{Merge Functions}

A first idea is that a conversation among partners depend on the number of entity cells that are in the mind-maps $M_{p}$ and $M_{p q}^{*}$. In that context, $M_{p}$ is the self mind-maps for the person $p$, whereas $M_{p q}^{*}$ is the mind-map of the person $p$ about the conversing partner $q$. The similarity of these two mind-maps is then the result of counting the corresponding entity cells contain the same information. A similarity is then given, if the final number of common entity cells reaches a certain threshold value. Furthermore, an improved version of this approach has been to take the activation status (and the corresponding connection status) into account. The similarity between the two mind-maps $M_{p}$ and $M_{p q}^{*}$ is then not only the 
pure number of common entity cells but moreover a weighted sum.

In general all the entity cells inside these two mind-maps are not often important or interesting to a person. Depending upon the conversational situations, some entity cells (and their associations) could be highly important - some remain negligible. We therefore consider the individual parallel universe, which is again categorised into the corresponding self-relevance $\rho^{M_{p}}$ and outer-relevance $\rho^{M_{p q}^{*}}$. Some relevance values are dynamic and may change over time. In general when the natural conversations are not influenced by some special situations, all the relevances (temporal patterns) are merged with the similar priority. Here, inside the mind-maps these special situations are defined by the highly activated entity cells (skeletons), which can also be considered as the the permanent impression (memory) about some events. Therefore the overall parallel universe does not exist in the beginning of the mind-maps life cycle, but gradually matures with various cognition capabilities - like these non-specified and specified mental representations and their corresponding merging situations.

\section{A. Merging Entity Cells}

So far, several alternatives have been identified, which do either use an a-priori knowledge or demand an ex-posteriori information. A first (and in our opinion the most appropriate) approach is to randomize each relevance value, which has the advantage that a-priori less/no efforts must be investigated. The belief that more important entity cells adapt to a higher relevance value (and less interesting entity cells to lower relevance values) can not be dismissed. The idea of initially differ between entity cells only considers for some specific events. Therefore in a simplistic approach, we firstly consider all entity cells with equal priority. Then the merge function $\mu\left(M_{p}, M_{p q}^{*}, t\right)$ is as follows:

$$
\mu\left(M_{p}, M_{p q}^{*}, t\right)=\sum_{i=1}^{k} \sum_{j=1}^{l} \frac{\left|\rho^{M_{p}}\left(e_{i}, t\right) \cap \rho^{M_{p q}^{*}}\left(e_{j}, t\right)\right|}{\left|\rho^{M_{p q}^{*}}\left(e_{j}, t\right)\right|}
$$

\section{B. Merging skeletons and Entity Cells}

The grade of accordance among conversational partners may certainly depend on the number of entity cells that are in the immortal mind-maps $M_{p}$ and $M_{p q}^{*}$, respectively, and a merge over weighted cells inside these mind-maps might eventually demonstrate how trustworthy a person is. However, these structures might be worthless or even less interesting in case that longer established skeletons exist in one of the memories. This observation follows the natural example, where a decision is partially influenced by recent events, ideals, and even longer established facts. With this, the following merge function $\mu^{\prime}$ between $M_{p}$ and $M_{p q}^{*}$ is an alternative to the previously defined function. Now, the self structural skeletons $\left(s_{i}\right)$, which exist inside the memory of the mind-maps, are considered. The time $t$ has been removed as such a parameter is senseless within immortal mind-maps.
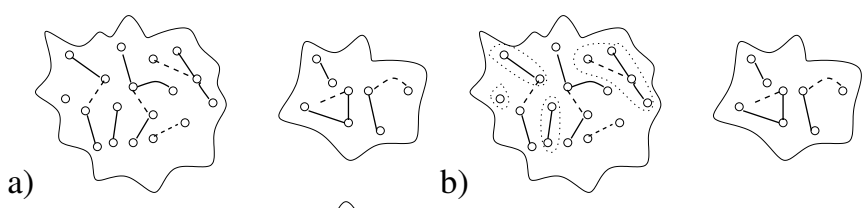

c)
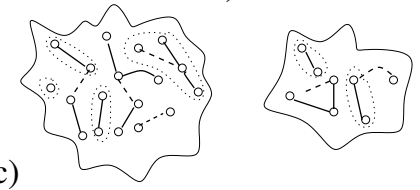

Fig. 5. Merge processes between a) entity cells, b) entity cells and skeletons, and c) skeletons and skeletons.

$$
\mu^{\prime}\left(M_{p}, M_{p q}^{*}\right)=\sum_{i=1}^{k} \sum_{j=1}^{l} \frac{\left|\rho^{M_{p}}\left(s_{i}\right) \cap \rho^{M_{p q}^{*}}\left(e_{j}\right)\right|}{\left|\rho^{M_{p q}^{*}}\left(e_{j}\right)\right|}
$$

\section{Merging skeletons}

A third merge function $\mu^{\prime \prime}$ concerns with longer established structures and not with recent events. For example, if Alice talks to $B o b$, then it is likely that Alice knows already Bob. It is therefore less of interest, what exactly $B o b$ says. However, there might be some indication that Bob 's comments may influence Alice's mind-map with respect to Bob. The merge function is then

$$
\mu^{\prime \prime}\left(M_{p}, M_{p q}^{*}\right)=\sum_{i=1}^{k} \sum_{j=1}^{l} \frac{\left|\rho^{M_{p}}\left(s_{i}\right) \cap \rho^{M_{p q}^{*}}\left(s_{j}\right)\right|}{\left|\rho^{M_{p q}^{*}}\left(s_{j}\right)\right|}
$$

$\mu^{\prime \prime}$ is not a time varying function as time has no influence for already structured skeletons $\left(s_{i}\right.$ and $s_{j}$ ) inside the mindmaps.

Figure 5 describes the merge for these functions. In a), we merge both inner cores of Alice's mind-maps (herself, left side) and her mind-maps about $B o b$ (right side), whereas b) represents the situation about strong temporal impression of Alice's mind-maps in the form of memory (rounded dotted shapes on the left side - skeletons) vs. the Alice's immortal mind-maps about Bob (right side), and c) the situation about the Alice's own strong beliefs (rounded dots on the left side) vs. the Alice's strong impressions about Bob (rounded dots on the right side).

\section{Discussion}

Currently, the explorative mind-map supports the creation of binary patterns, which are associatively connected between two adjacent cells: in case that a signal $\{A, B, C\}$ arrives, a full-connected cell clump made of $A, B$, and $C$ is sent to the main memory trying to merge it with existing cells. But all results of this merge remain binary. An extension therefore is to understand temporal signals as n-ary patterns: these are formed by several entity cells by an associated connectivity. Here, the formation of the patterns is a bottom-up process that evolves several learning steps. In this regard, the input 
signals are decomposed to frames, where each frame is to be understood as a sub-set of a sentence (a phrase) or even the sentence itself. If at a particular time the overall text input stream is considered as to be the content that is received from the conversational partner, then each sentence can be distinguished by such a frame. The filtration (extraction) of individual frames influences the entity cells and their connectivity: the cells of a newly filtered frame compare its immediate predecessor frame with the beginner frame of the same content (in an iterative way). On the one hand, this results in novel patterns, and on the other in an update of activation values after each iteration. If there exist no common cells, then obviously more than one patterns will be obtained.

Consider the example of a natural text stream that is received by Alice's conversational partner $(B o b)$. It influences the desired pattern formation inside Alice's mind-map concerning Bob:

Bob: I have a football.

Bob: Footballs are round.

There exist two frames: after having processed the first frame, the single entity cell football comes out as the main subject. This is filtered with an activation value 1 due to its first occurrence. The second frame owns then two entity cells football and round, respectively. Here, the connection between these entity cells is the main learning outcome (subject) of this frame. It activates the new pattern with an activation value of 1 with respect to the connection between the entity cells. Let us now assume that in the next communication phase the followoing input signal is quoted by Bob:

Bob: I have mentioned about round shape.

Bob: Round shapes are very common in the universe.

Bob: For example, shape of most of stars are round.

Then, the new n-ary patterns are obtained at the same time. The connectivity is clearly based on the relation among the n-ary pattern; the activation values for the individual $n$ ary patterns are updated stepwise. Finally the extracted n-ary patterns are merged with the extracted n-ary pattern of its previous content based on the common subject cell round. If there are no common cells between two n-ary patterns, then both patterns are kept separately inside the mind-map memory. In this way both Alice's and Bob's own mind-maps have been established. Also when the following continuous conversation between Alice and $B o b$ takes place then both mind-maps are taken into account:

Bob: The sun is shining, what a beautiful day. Alice: The sun is very hot.

Bob\}: This is right, but I like sunny days.

Bob's affirmation is inherited to Alice's mind-map and the entity cell day is activated double and that the connection between day and sunny has been strengthened.

\section{COnClusions}

In this paper, we have concerned with the nature-inspired processing of data input streams through explorative mindmaps. In the presence of a natural conversation, the presented model takes advantage of an associative management structure, a consistency of the cell membrane and the temperature inside the mind-map. Moreover, we have shown a merge of existing, but independent, conversational skeletons and described the mind-map model in detail and figured out a diverse number of merge functions and transfer rates. So far, we have applied explorative mind-maps in various situations [10], for example as backbone in a intrusion detection system or as query manager in an information retrieval system SEREBIF.

With the presented extended model, however, we follow the path of recommending trust in the presence of a conversation. Having two mind-maps $M_{p}$ and $M_{p q}^{*}$ for a person $p$ and the minimum number $k$ and $l$ of entity cells, which share the relevance values $\rho^{M_{p}}\left(e_{i}, t\right)$ and $\rho^{M_{p q}^{*}}\left(e_{j}, t\right)$, respectively, then a trust function $\operatorname{gtrust}()$ may be given as

$$
\operatorname{gtrust}\left(M_{p}, M_{p q}^{*}, t\right)= \begin{cases}y e s, & \mu\left(M_{p}, M_{p q}^{*}, t\right) \geq \alpha \\ n o, & \text { else }\end{cases}
$$

The trust threshold $\alpha$ is given individual to each conversing partner. Here the decision on how to trust the conversational partner is binary but also the trust level can be quantified with the measured value $\mu$. With this as well as other with other model-related aspects, we are optimistic to come up with a trust decision system.

\section{ACKNOWLEDGEMENT}

This work has been done at the MINE research group, which is a member of the Interdisciplinary Laboratory for Intelligent and Adaptive Systems (ILIAS), University of Luxembourg.

\section{REFERENCES}

[1] J. L. Elman, E. A. Bates, M. H. Johnson, A. Karmiloff-Smith, D. Parisi, and K. Plunkett, (1996), Re-thinking Innateness: A connectionist perspective on development. MIT Press, Cambridge, MA, 1995.

[2] S. Franklin, Articial Minds. MIT Press, Cambridge, MA.

[3] C. Kemke, Generative connectionist parsing with dynamic neural networks. In Proceedings of the $2^{\text {nd }}$ Workshop on Natural Language Processing and Neural Networks, 2001.

[4] G. F. Luger, P. Johnson, C. Stern, and J. E. Newman, Cognitive Science: The Science of Intelligent Systems. Academic Press, 1994.

[5] G. F. Marcus, The Algebraic Mind: Integrating Connectionism and Cognitive Science (Learning, Development, and Conceptual Change). MIT Press, Cambridge, MA, 2001.

[6] K. Plunkett, E. T. Rolls, and P. McLeod, Introduction to Connectionist Modelling of Cognitive Processes. Oxford University Press, 1998.

[7] T.A. Polk, and C. M. Seifert, Cognitive Modeling. The MIT Press, 2002.

[8] J. Poray and C. Schommer (2009), A cognitive mind-map framework to foster trust. In The $5^{\text {th }}$ International Conference on Natural Computation (ICNC09).

[9] D. E. Rumelhart, J.L. McClelland, and the PDP Research Group, Parallel Distributed Processing: Explorations in the Microstructure of Cognition. Volume 1: Foundations. MIT Press, Cambridge, MA, 1986.

[10] C. Brucks, M. Hilker, C. Schommer, C. Wagner, R. Weires, Symbolic Computing with Incremental Mindmaps to Manage and Mine Data Streams - Some Applications. Proceedings of the $4^{\text {th }}$ International Workshop on Neural-Symbolic Learning and Reasoning (NeSy '08), 2008.

[11] P. Thagard, Mind: Introduction to Cognitive Science. The MIT Press, 2005 . 